\title{
Loss of BICD2 in muscle drives motor neuron loss in a developmental form of spinal muscular atrophy
}

\author{
Alexander M. Rossor ${ }^{1 *}$ (D), James N. Sleigh ${ }^{1,2}$, Michael Groves ${ }^{1}$, Francesco Muntoni ${ }^{3}$, Mary M. Reilly ${ }^{1}$, \\ Casper C. Hoogenraad ${ }^{4}$ and Giampietro Schiavo ${ }^{1,2,5^{*}}$ (D)
}

\begin{abstract}
Autosomal dominant missense mutations in BICD2 cause Spinal Muscular Atrophy Lower Extremity Predominant 2 (SMALED2), a developmental disease of motor neurons. BICD2 is a key component of the cytoplasmic dynein/dynactin motor complex, which in axons drives the microtubule-dependent retrograde transport of intracellular cargo towards the cell soma. Patients with pathological mutations in BICD2 develop malformations of cortical and cerebellar development similar to Bicd2 knockout (-/-) mice. In this study we sought to re-examine the motor neuron phenotype of conditional Bicd $2^{-1-}$ mice. Bicd $2^{-1}$ mice show a significant reduction in the number of large calibre motor neurons of the $L 4$ ventral root compared to wild type mice. Muscle-specific knockout of Bicd2 results in a similar reduction in $L 4$ ventral axons comparable to global Bicd2 $2^{-1-}$ mice. Rab6, a small GTPase required for the sorting of exocytic vesicles from the Trans Golgi Network to the plasma membrane is a major binding partner of BICD2. We therefore examined the secretory pathway in SMALED2 patient fibroblasts and demonstrated that BICD2 is required for physiological flow of constitutive secretory cargoes from the Trans Golgi Network to the plasma membrane using a VSV-G reporter assay. Together, these data indicate that BICD2 loss from muscles is a major driver of non-cell autonomous pathology in the motor nervous system, which has important implications for future therapeutic approaches in SMALED2.
\end{abstract}

Keywords: Spinal muscular atrophy, SMALED2, Hereditary motor neuropathy, BICD2, DYNC1H1, Muscle

\section{Introduction}

Spinal Muscular Atrophy Lower Extremity Predominant (SMALED) is a disease of lower motor neurons, principally affecting the lower limbs. Affected individuals often present at birth with contractures of the lower limbs. Autosomal dominant missense mutations in DYNC1H1 and BICD2 are the only two known genetic causes of SMALED and determine two indistinguishable forms of this disease [1-5].

DYNC1H1 encodes the cytoplasmic dynein heavy chain, a key component and force-generating subunit of the dynein/dynactin retrograde transport complex. This complex

\footnotetext{
* Correspondence: a.rossor@ucl.ac.uk; giampietro.schiavo@ucl.ac.uk ${ }^{1}$ UCL Queen Square Institute of Neurology, University College London, Queen Square, London WC1N 3BG, UK

Full list of author information is available at the end of the article
}

is responsible for the transport of intracellular cargoes towards the minus-end of microtubules located in the cell soma [6]. In contrast, anterograde transport towards the positive end of microtubules is driven by the kinesin family of motor proteins [7].

Motor neurons have extremely long axons, which make them preferentially susceptible to deficits in axonal transport [8]. It has long been thought that mutations in BICD2 and DYNC1H1 impair axonal transport leading to motor neuron degeneration. This is supported by work in Drosophila demonstrating a reduction of the in vitro run length in flies expressing disease-causing mutant DYNC1H1 compared to wild type [9]. This conclusion has been brought into question by the contrasting findings that several disease-causing mutations in BICD2 increase its binding 
affinity to the dynein/dynactin complex resulting in an increase in run length [10].

BICD2 is a cargo adaptor protein, comprising a $\mathrm{N}$ terminal region that mediates binding to the dimerization domain of DYNC1H1 and a C-terminal cargo binding domain [11]. Missense mutations throughout BICD2 have been shown to cause SMALED2 [12], yet the disease mechanism is unclear. Mutations in the N-terminal domain increase the affinity to the dynein complex [10], whereas the p.Glu774Gly mutation in the C-terminal domain has no effect on DYNC1H1 binding, but disrupts the interaction with its cargo Rab6, causing a loss of function phenotype [5]. As BICD2 forms dimers, such mutations may also impair the cargo binding ability of wild type/mutant BICD2 heterodimers, leading to near complete loss of function as opposed to haploinsufficiency. A loss of function effect in SMALED2 was thought to be unlikely as the $B i c d 2^{-/-}$ mouse was originally reported to lack a motor phenotype [13]. However, recent studies of SMALED2 patients with severe mutations in $B I C D 2$ have revealed additional cortical and cerebellar phenotypes similar to the $B i c d 2^{-/-}$mouse. These findings suggest that pathological BICD2 mutations may indeed induce a loss of function [14]. In this study, we sought to re-examine the motor neuron phenotype in the Bicd $2^{-/-}$mouse to conclusively assess the molecular basis of the pathomechanism of SMALED2.

\section{Materials and methods \\ Animals}

Mice homozygous for an allele in which the loxP sequence is inserted into intron 1 and the 3'UTR of the endogenous Bicd2 gene [13] were crossed with heterozygous deleterCre mice (C57BL/6NTac-Gt(ROSA)26Sor ${ }^{\text {tm16(cre)Arte) [15] }}$ to generate $\mathrm{Bicd} 2$ heterozygous knockout mice expressing a Cre-recombinase transgene. These mice were subsequently crossed with $\mathrm{C} 57 \mathrm{Bl} / 6 \mathrm{~J}$ mice to generate $\mathrm{Bicd} 2^{+/-}$ mice with no Cre recombinase transgene. Genotypes of mice were determined by PCR of ear clip DNA (P1 = AATGGAGAAGATCTCATCTTGGCAGG, P2 = GTGT AGCACTTCAGGAACATCCATGC, P3 = TGTCAGCAA ACTCCATCTCTAGCCTC, P261 = CGGCGGCATCAGA GCAGCCGATTG).

To generate muscle-specific Bicd 2 knockout mice, homozygous Bicd $2^{\text {loxP/loxP }}$ mice $(\mathrm{C} 57 \mathrm{Bl} / 6 \mathrm{~J})$ were crossed with knockin MyoD-Cre mice (FVB.Cg-Myod1 $1^{\text {tm2.1(icre)Glh } / \text {, }}$, Jackson laboratory) [16] and back-crossed to the original Bicd $2^{\text {loxP/loxP }}$ progeny for six generations to produce a congenic strain. To generate motor neuron-specific Bicd2 knockout mice, homozygous Bicd2 ${ }^{\text {loxP/loxP }}$ mice (C57Bl/6 J) were crossed with ChAT-IRES-Cre knock-in mice (B6;

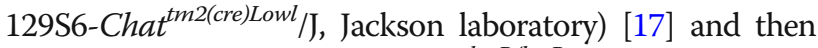
back-crossed to the original $B i c d 2^{\operatorname{loxP} / \operatorname{lox} P}$ progeny to generate a homozygous Bicd2 $2^{\text {loxP/loxP }}$ background.

\section{Histopathology}

Mice were terminally anaesthetised with intraperitoneal injection of pentobarbitone followed by thoracotomy and transcardial perfusion with $10 \mathrm{ml} 0.9 \% \mathrm{NaCl}$ followed by $20 \mathrm{ml} \mathrm{4 \%}$ paraformaldehyde (PFA, Fisher Scientific) in phosphate buffered saline (PBS). L4 DRG and L4 dorsal and ventral roots were dissected from PFA-fixed mice and post-fixed in PFA/glutaraldehyde buffer at $4{ }^{\circ} \mathrm{C}(20 \mathrm{ml}$ 0.9\% saline, $10 \mathrm{ml} \mathrm{10 \%} \mathrm{PFA,} 10 \mathrm{ml} \mathrm{10 \%} \mathrm{glutaraldehyde}$ (Sigma-Aldrich), 20\% dextran (20,000 MW, SigmaAldrich) made up to $100 \mathrm{ml}$ with $0.1 \mathrm{M}$ PIPES-NaOH, $\mathrm{pH}$ 7.6, for $24 \mathrm{~h}$ before fixation in $1 \%$ osmium tetroxide (Agar Scientific) and processing into araldite CY212 epoxy resin (Agar Scientific) through graded alcohols and propylene oxide solutions using a standard protocol. Semi-thin sections $(0.8 \mu \mathrm{m})$ were cut on an Ultracut $\mathrm{E}$ ultramicrotome (Leica), stained with $1 \%$ toluidine blue containing $1 \%$ bo$\operatorname{rax}(\mathrm{BDH})$, and examined with a Leica light microscope using an oil immersion 100x lens. Images were generated by image stitching using HUGIN-panorama photo stitching. Axon diameter was calculated using an in-house semi-automated thresholding programme using Definiens image analysis software.

\section{Immunohistochemistry (For a summary of antibodies see Table 1) \\ Muscle fibre subtype and area quantification}

P21 mice were euthanized by cervical dislocation and skeletal muscles (gastrocnemius) were dissected and snap frozen in liquid nitrogen-cooled isopentane and stored at $-80^{\circ} \mathrm{C}$ for cryosectioning. Immunofluorescent staining was carried out on $10 \mu \mathrm{m}$ frozen sections. Sections were blocked in PBS $0.2 \%$ Triton X-100, 5\% goat serum for $1 \mathrm{~h}$. Primary and secondary antibodies were diluted in PBS $0.2 \%$ Triton X-100, $2 \%$ goat serum. Primary antibodies: Type 1 BA-D5 (mouse IgG2b) DSHB (1 in 100), Type IIA SC-71 (mouse IgG1) DSHB (1 in 100), Type IIB BF-F3 (mouse IgM) DSHB (1 in 100) and laminin (rabbit) L9393 Sigma-Aldrich (1 in 100) were incubated for $1 \mathrm{~h}$ at $37^{\circ} \mathrm{C}$. Sections were then washed three times with PBS followed by goat anti-mouse IgG2b conjugated to AlexaFluor488, goat anti-mouse IgG1 conjugated to AlexaFluor647, goat anti-mouse IgM conjugated to AlexaFluor568 and goat anti-rabbit conjugated to AlexaFluor410 (all from Life Technology, 1:500). Secondary antibodies were incubated for $30 \mathrm{~min}$ at $37^{\circ} \mathrm{C}$ and washed three times with PBS. Cover slips were mounted on stained sections using Dako fluorescent mounting media and dried for $48 \mathrm{~h}$. Z-stack stitched images were acquired using a Zeiss LSM 710 confocal microscope using previously described parameters [18]. Quantification of the fibre number, area and type were performed using the muscle-J automated freeware and Image J as previously described [18]. 
Table 1 Primary and secondary antibodies

\begin{tabular}{|c|c|c|c|}
\hline Epitope & Antibody & Concentration & Species \\
\hline Type 1 muscle fibres & BA-D5 (DSHB) & 1 in 100 & Mouse $\lg G 2 \mathrm{~b}$ \\
\hline Type IIA muscle fibres & SC-71 (DSHB) & 1 in 100 & Mouse lgG1 \\
\hline Type IIB muscle fibres & BF-F3 (DSHB) & 1 in 100 & Mouse IgM \\
\hline Laminin & L9393 (Sigma-Aldrich) & 1 in 100 & Rabbit \\
\hline Mouse lgG2b & AlexaFluor 488 (Life Sciences) & 1 in 500 & Goat \\
\hline Mouse lgG1 & AlexaFluor 647 (Life Sciences) & 1 in 500 & Goat \\
\hline Mouse lgM & AlexaFluor 568 (Life Sciences) & 1 in 500 & Goat \\
\hline Rabbit lgG & AlexaFluor 410 (Life Sciences) & 1 in 500 & Goat \\
\hline Mouse lgG & AlexaFluor 488 (Life Sciences) & 1 in 250 & Goat \\
\hline $2 \mathrm{H} 3$ & DSHB (Supernatant) & 1 in 50 & Mouse lgG1 \\
\hline \multirow[t]{2}{*}{ SV2 } & DSHB (Supernatant) & 1 in 100 & Mouse $\lg G 1$ \\
\hline & $\begin{array}{l}\text { Tetramethylrhodamine-bungaratoxin } \\
\text { (-BTX, Cambridge Bioscience BT00012) }\end{array}$ & $1.5 \mu \mathrm{g} / \mathrm{ml}$ & \\
\hline NF200 & N0142, SIGMA & 1 in 500 & Mouse lgG \\
\hline Peripherin & Merck Millipore, AB1530 & 1 in 500 & Rabbit lgG \\
\hline VSV-G & 8G5F11, Kerafast & 1 in 2000 & Mouse lgG \\
\hline
\end{tabular}

DSHB Developmental Studies Hybridoma Bank

\section{NMJ analyses}

Muscles were processed for immunohistochemistry and NMJ phenotypes scored as previously described [19]. 100 NMJs were scored per mouse for poly-innervation and occupancy counts, whilst 16-20 NMJs were used for area measurements.

\section{Muscle spindle assessment}

Soleus muscles were dissected and processed, and spindles analysed as described previously with minor modifications [20]. Briefly, soleus muscles were dissected then fixed overnight in 4\% PFA in PBS prior to overnight equilibration in $20 \%(\mathrm{w} / \mathrm{v})$ sucrose in PBS before freezing in Tissue-Tek O.C.T. (Sakura Finetek). $20 \mu \mathrm{m}$ transverse serial sections throughout the entire muscle were cut onto three parallel slides for immunohistochemistry and analyses using an OTF Cryostat (Bright Instruments).

\section{DRG dissection and staining}

Mice were perfused with 4\% PFA in PBS before L4 DRG were dissected as previously described [21]. DRG were sectioned and stained as previously published [20]. Briefly, DRG were post-fixed overnight in 4\% PFA in PBS, before embedding in Tissue-Tek O.C.T., and sectioning at $10 \mu \mathrm{m}$ across four parallel polysine-coated slides (VWR, 6310107) with an OTF Cryostat. DRG sections were permeabilised for $30 \mathrm{~min}$ in PBS containing 0.3\% Triton X-100 and blocked for $30 \mathrm{~min}$ in $10 \%$ bovine serum albumin (BSA) and $0.3 \%$ Triton X-100 in PBS, before probing overnight at $4{ }^{\circ} \mathrm{C}$ with primary antibodies (1:500 mouse anti-NF200 [N0142; Sigma-Aldrich] and 1:500 rabbit anti-peripherin (AB1530; Merck Millipore) in blocking solution. Sections were then washed with PBS for 30 min, probed for $2 \mathrm{~h}$ with secondary antibodies (1:1000) in PBS, before washing in PBS, flooding with fluorescence mounting medium (S3023; Dako) and covering with a $22 \times 50 \mathrm{~mm}$ cover glass (VWR).

\section{VSVG assay}

Frozen vials of human skin fibroblasts were thawed and grown in T75 flasks for seven days in DMEM media supplemented with $20 \%$ foetal calf serum and L-glutamine (all from Gibco). Cells were trypsinised and plated onto $13 \mathrm{~mm}$ untreated glass coverslips (50,000 per coverslip). After $24 \mathrm{~h}$, fibroblasts were transfected with a GFP-VSV$\mathrm{G}$ ts045 plasmid using Lipofectamine 3000 (Invitrogen) as per manufacturer's instructions and grown at $37^{\circ} \mathrm{C}$ (VSV = vesicular stomatitis virus). After $8 \mathrm{~h}$, cells were incubated at $40{ }^{\circ} \mathrm{C}$ for $14 \mathrm{~h}$ before being transferred to an incubator at $32^{\circ} \mathrm{C}$. Cells were fixed at 0, 60, 120, 180, 240 and $300 \mathrm{~min}$ after incubation at $32^{\circ} \mathrm{C}$ in PFA $4 \%$ in PBS. Non-permeabilised cells were then incubated with PBS $1 \%$ BSA for 15 min prior to overnight incubation with mouse anti-VSV-G [8G5F11; Kerafast] antibody $(1: 2000)$ at $4{ }^{\circ} \mathrm{C}$. Cells were washed three times with PBS before incubation with a donkey anti-mouse AlexaFluor568 secondary antibody (Life Technology, 1:500) for $1 \mathrm{~h}$ at room temperature. Cells were then washed, stained with DAPI (1:2000) and coverslips mounted and fixed with DAKO fluorescent mounting media. Ten fibroblasts per condition were imaged with a Zeiss LSM 510 confocal microscope and the ratio of the fluorescence between plasma membrane VSV-G (594 nm excitation) to the total VSV-G (GFP signal, $488 \mathrm{~nm}$ excitation) was quantified as previously described [22]. 


\section{Brefeldin a assay}

Human fibroblasts were cultured as previously described above, but were plated on $20 \mathrm{~mm}$ coverslips in 6 well plates at a density of 100,000 per well. After $24 \mathrm{~h}$ in culture, cells were transfected with a plasmid encoding human galactosyltransferase tagged with GFP (GT-GFP) (kind gift from Masayuki Murata, Tokyo). 24h posttransfection, cells were imaged using a Zeiss LSM 710 confocal microscope. Briefly, cells were imaged at $37^{\circ} \mathrm{C}$, $5 \% \mathrm{CO}_{2}$ with a 40x objective. The Golgi apparatus was identified by GFP staining. Brefeldin A ((BFA), $10 \mu \mathrm{g} / \mathrm{ml}$ final concentration; Sigma-Aldrich) was re-suspended in DMEM, 10\% FCS, 20 mM HEPES-NaOH (final concentration), $\mathrm{pH} 7.3$, and added to the coverslip at time 0 . Time-lapse imaging at $5 \mathrm{~s}$ intervals was carried out and the time recorded to the beginning and end of the 'Golgi blush', defined as the stereotypical changes in Golgi morphology leading to its resorption into the ER.

\section{Statistical analysis}

Data were assumed to be normally distributed unless evidence to the contrary was provided by the D'Agostino and Pearson omnibus normality test. Data were statistically analysed using an unpaired $t$ test, Mann-Whitney $U$-test, or one-way ANOVA with post-hoc Dunnett's test against a control mean. GraphPad Prism 6 software was used for all statistical analyses. Means \pm standard error of the mean (SEM) were plotted for all graphs.

\section{Results and discussion}

\section{Bicd2 $2^{-/-}$mice display a significant motor neuron loss}

Due to the similarities between severe SMALED2 patients and Bicd $2^{-/-}$mice $[13,14]$ and the observation that missense mutations in the cargo binding domain of BICD2 impair Rab6 binding with no effect on dynein binding [5], we predicted a loss of function pathomechanism in SMALED2. Following this hypothesis, loss of BICD2 function should result in motor neuron loss. We therefore re-examined the motor neuron phenotype of $B i c d 2^{-/-}$mice by examining the L4 dorsal and ventral nerve roots in the spinal cord of wild type and knockout mice at postnatal day 21 (p21). We were unable to examine these mice at later time points as knockout mice die by four weeks of age from obstructive hydrocephalus [13]. The L4 nerve root was chosen as it is the largest lumbar nerve root. At p21, there is a significant reduction in the total number of motor axons in $B i c d 2^{-/-}$mice compared to wild type and this is restricted to a subpopulation of motor axons with a diameter of $2.5-4 \mu \mathrm{m}$ (Fig. 1a-c; Bicd $2^{+/+}$mean $=926 \pm 22.7$ (SEM, $n=7)$, Bicd $2^{-/-}=806 \pm 25.0(\mathrm{n}=7)$, unpaired $t$ test, $p=0.0041)$. In contrast, there was no reduction in the number of L4 dorsal root sensory axons between wild type $(1778 \pm 35.8 ; n=6)$ and $B i c d 2^{-/-}(1792 \pm 60.4$; $\mathrm{n}=6$ ) mice (unpaired t-test, $p=0.85$ ) (Fig. 1d-f). The lack of a sensory phenotype was corroborated by further analyses of the percentage of medium-to-large, NF200 ${ }^{+}$neurons, and small peripherin ${ }^{+}$neurons, in the L4 dorsal root ganglia (DRG), which did not reveal any difference between wild type and Bicd $2^{-/-}$mice (Supplementary Figure 1).

\section{Loss of motor neurons in Bicd2 $2^{-/-}$mice is caused by a muscle non-cell autonomous process}

The cerebellar hypoplasia phenotype observed in $B i c d 2^{-/-}$mice is due to a non-cell autonomous process arising in Bergmann glia [13]. Therefore, we hypothesised that the motor neuron loss we observed in the same knockout mice may also be due to a non-cell autonomous mechanism, but in this instance, arising from muscle tissue, which has previously been shown to have important trophic support functions for motor neurons [23]. According to the neurotrophin hypothesis, during early development, an excess of motor neurons reach their target muscle and compete for muscle-secreted survival factors (e.g. neurotrophins) [24]. A stochastic process of programmed cell death then follows, as those motor neurons that receive insufficient neurotrophins undergo apoptosis. To test this hypothesis, we generated mice in which Bicd2 is selectively knocked out only in skeletal muscle. Homozygous Bicd2 mice, in which the endogenous Bicd2 allele is flanked by intronic loxP sequences [13], were crossed with mice expressing crerecombinase driven by the endogenous Myod promoter to generate mice lacking both Bicd2 alleles in muscle tissue alone $[13,16]$. These mice had a normal life span and no obvious gait abnormality. Analyses of these mice at p21 shows a significant reduction in motor axons in the $\mathrm{L} 4$ ventral nerve root compared to wild type mice (Fig. 2a and c; Bicd $2^{+/+}=928 \pm 24(n=7)$, Myod-Cre $=$ $817 \pm 39(\mathrm{n}=7)$, one-way ANOVA $p=0.019$, Dunnett's $t$-test, $p=0.034)$. Furthermore, the subpopulation of motor axons affected is the same as the Bicd $2^{-1-}$ mouse (2.5-4.4 $\mu \mathrm{m}$ diameter). A small reduction in the number of L4 motor axons between wild type $(928 \pm 24)$ and homozygous Bicd $2^{\text {loxP/loxP }}(888 \pm 23, n=7$; Dunnett's $t$ test, $p=0.705)$ mice was detected, but it did not reach statistical significance (Fig. 2a). To confirm that the loss of motor axons in $B i c d 2^{-/-}$mice was solely due to loss of Bicd2 in muscle, we generated mice lacking Bicd2 in motor neurons. Homozygous Bicd $2^{\text {loxP/loxP }}$ mice were crossed with mice expressing cre-recombinase driven by the endogenous ChAT promoter to generate mice lacking both Bicd2 alleles selectively in motor neurons. Analysis of the number of L4 motor axons in these genetically modified mice at p21 revealed no difference compared to Bicd $2^{+/+}$mice (Fig. 2a and b; Bicd $2^{+/+}=$ $928 \pm 24 ; \quad \mathrm{n}=7 ; \quad$ ChAT-Cre $=924 \pm 23, \quad n=3 ; \quad$ one-way ANOVA $p=0.019$, Dunnett's $t$-test, $p=0.939)$. 


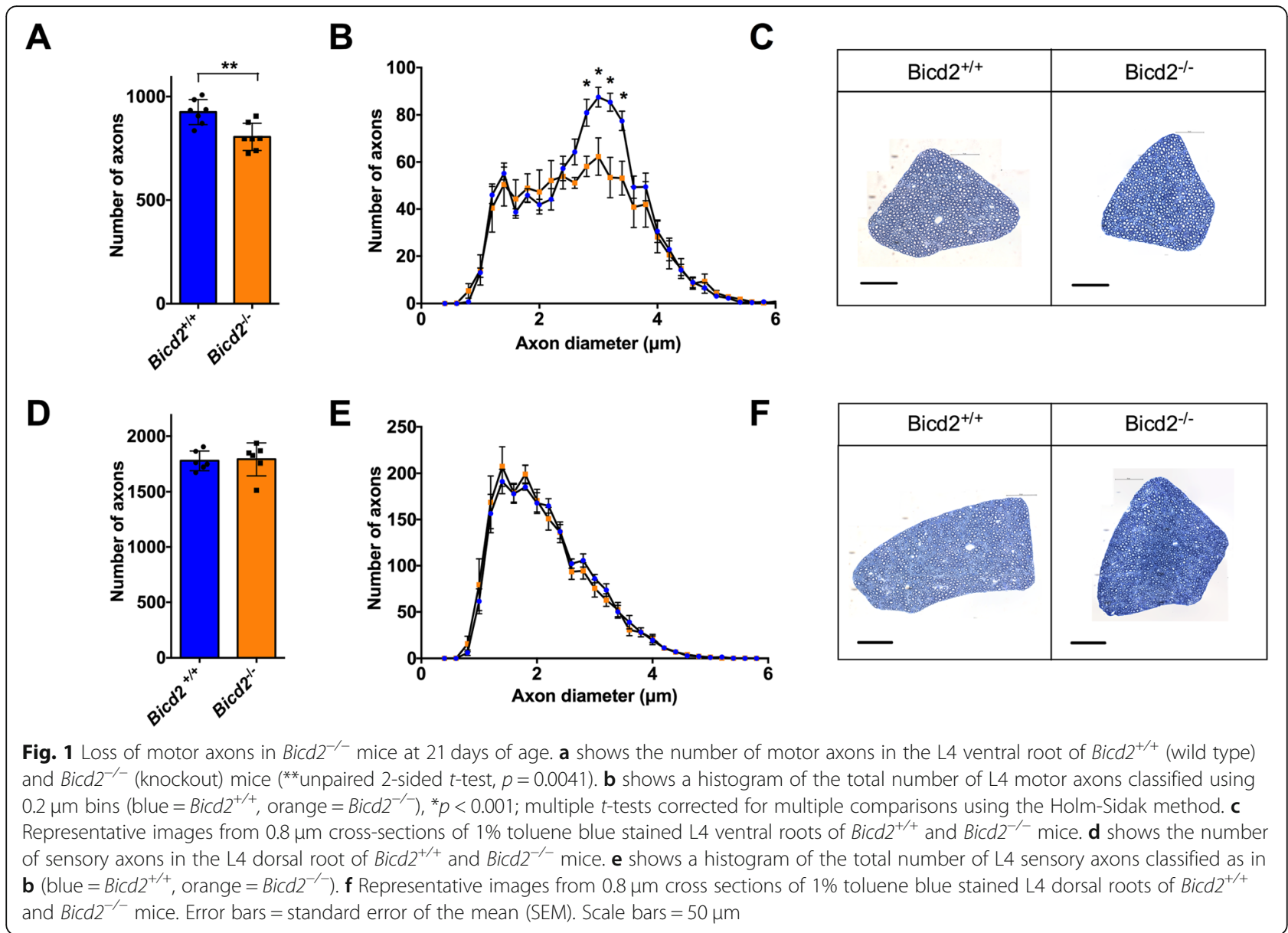

A similar analysis performed on the legs at odd angles mouse' (loa) strain, which bear a missense mutation in Dync1h1 (a binding partner of Bicd2), showed at a later time point of six weeks a reduction in small diameter motor axons, which were assumed to be gamma motor neurons $[25,26]$. We therefore repeated this analysis in Myod-Cre mice at six weeks of age. A comparison of the axon diameter cumulative distribution curves shows a loss of motor axons in mice with muscle-specific knockout of Bicd2, revealing a decrease in a subset of motor axons of smaller diameter in the putative range of gamma motor neurons (Fig. 2d). Myod-Cre mice showed no obvious behavioural phenotype which would be in concordance with only a $10 \%$ loss of motor neurons.

\section{Active denervation is absent in Bicd2 ${ }^{-/-}$mice}

Next, we sought to determine whether the loss of motor neurons was due to active degeneration of motor axons. Morphological analysis of semi-thin ventral root sections revealed no acute axon degeneration profiles (Fig. 1c). We therefore analysed the neuromuscular junctions (NM)s) from two different muscles (lumbricals and flexor digitorum brevis (FDB) of the hind paw). Wholemount preparations of these two thin muscles were chosen due to reliable quantification of NMJ degeneration without the need for sectioning [19]. Interestingly, all muscles showed a full NMJ innervation pattern, suggesting that at the time of examination, there was no significant denervation (Fig. 3a and c). Furthermore, there was no evidence to indicate that the postnatal developmental process of synapse elimination at the NMJ was affected in Bicd $2^{-/-}$mice (Fig. 3b). In contrast, the NMJ area of the hindfoot lumbrical and FDB muscles was significantly smaller in Bicd $2^{-/}$compared to wild type mice (Fig. 3d). The significance of this is not clear but may be a consequence of the significant reduction in overall size of Bicd $2^{-/-}$mice and their muscle mass compared to wild type animals (Supplementary Figure 2A and C).

\section{Muscles in Bicd $2^{-/}$mice display loss of muscle spindles and gamma motor neurons, but no gross morphological changes}

Patients with SMALED2 show muscle biopsy abnormalities indicative of both neurogenic and myopathic processes [27]. We therefore examined the gastrocnemius muscle of wild type and $B i c d 2^{-/-}$mice, but did not identify any consistent differences on H\&E-stained muscle or on electron 



C
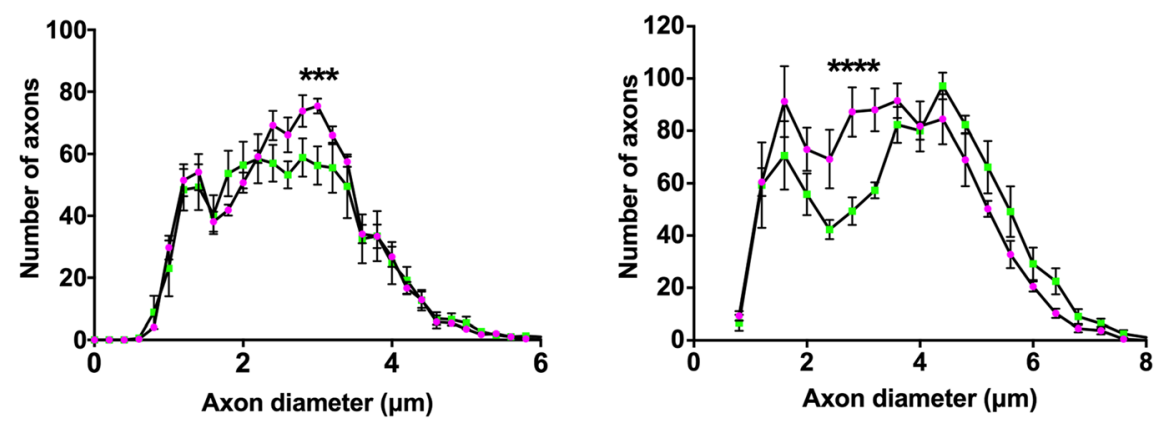

Fig. 2 Motor axon loss in muscle specific Bicd2 knockout mice. a shows the total number of axons at 21 days of age in Bicd2 ${ }^{+/+}$mice (blue), Bicd2 ${ }^{\text {loxP/loxP }}$ mice in which both Bicd2 alleles are flanked by loxP sites (pink), mice with motor neuron specific Bicd2 knockout (ChAT-Cre, red), mice with muscle-specific Bicd2 knockout (Myod-Cre, green), and Bicd2 ${ }^{-/-}$mice (orange). A significant reduction in the total number of $L 4$ ventral axons was found in Myod-Cre and Bicd2 ${ }^{-/-}$mice compared to wild type (one-way ANOVA $p=0.0187$, Dunnett's 2-sided $t$-test with Bicd2 $2^{+/+}$as control, ${ }^{* *} p=0.019,{ }^{*} p=0.034$ ). $\mathbf{b}$ shows a histogram of the total number of $L 4$ ventral axons at 21 days of age classified using $0.2 \mu \mathrm{m}$ bins (pink $=$ Bicd2 $2^{\text {loxP/loxP }}$, red $=$ ChAT-Cre). $\mathbf{c}$ shows a histogram of the total number of $L 4$ motor axons at 21 days of age classified using $0.2 \mu \mathrm{m}$ bins (pink= Bicd2 ${ }^{\text {loxP/loxP }}$, green = Myod-Cre); multiple $t$-tests corrected for multiple comparisons using the Holm-Sidak method, ${ }^{* * *} p=0.0009$. $\mathbf{d}$ shows a histogram of the total number of L4 motor axons at 42 days of age classified using $0.4 \mu \mathrm{m}$ bins (pink $=$ Bicd2 $2^{\text {loxploxp }}(n=4)$, green $=$ Myod-Cre $(n=$ 6); multiple t-tests corrected for multiple comparisons using the Holm-Sidak method, ${ }^{* * * *} p=0.0002$ ). Error bars $=$ SEM

microscopy (Supplementary Figure 3A-D) other than a non-significant reduction in muscle fibre diameter in Bicd2 $2^{-1-}$ compared to Bicd2 ${ }^{+/+}$mice and a significant reduction in total muscle (and body) mass (Supplementary Figure 2A,C\&D).

Motor neuron subtypes may be subdivided according to the fibre type they innervate [24]. For example, 'fast' alpha motor neurons innervate types IIa, IIb and IIx muscle fibres, whereas slow motor neurons innervate type 1 muscle fibres. We therefore examined the proportion of muscle fibres of the L4 innervated gastrocnemius muscle in wild type and $B i c d 2^{-/-}$mice, but we found no difference (Supplementary Figure 2F), indicating that Bicd 2 ablation does not induce a significant muscle fibre switch.

As shown in Fig. 2c and d, at both three and six weeks of age, the population of motor axons lost in the L4 ventral roots of mice in which Bicd2 has been selectively knocked out in muscle falls within the $2.5-4 \mu \mathrm{m}$ range also seen in $\mathrm{Bicd}^{-/-}$mice. The lack of denervation of NMJs or switch in muscle fibre types, suggests that alpha motor neurons are not affected. At six weeks of age, based on axon diameter, the loss of axons falls within the (Fig. 2d) presumed gamma motor neuron population. This is the same population of motor neurons that is lost in mice with missense mutations in Dync1h1 [26], which model SMALED in humans. The selective loss of gamma motor neurons in these two models suggests a deficit in muscle spindle derived GDNF secretion as GDNF knockout mice show isolated gamma motor neuron loss [28]. Gamma motor neurons represent approximately 30\% of motor neurons innervating muscle, but, unlike large calibre alpha motor neurons, they do not form NMJs with skeletal muscle fibres 


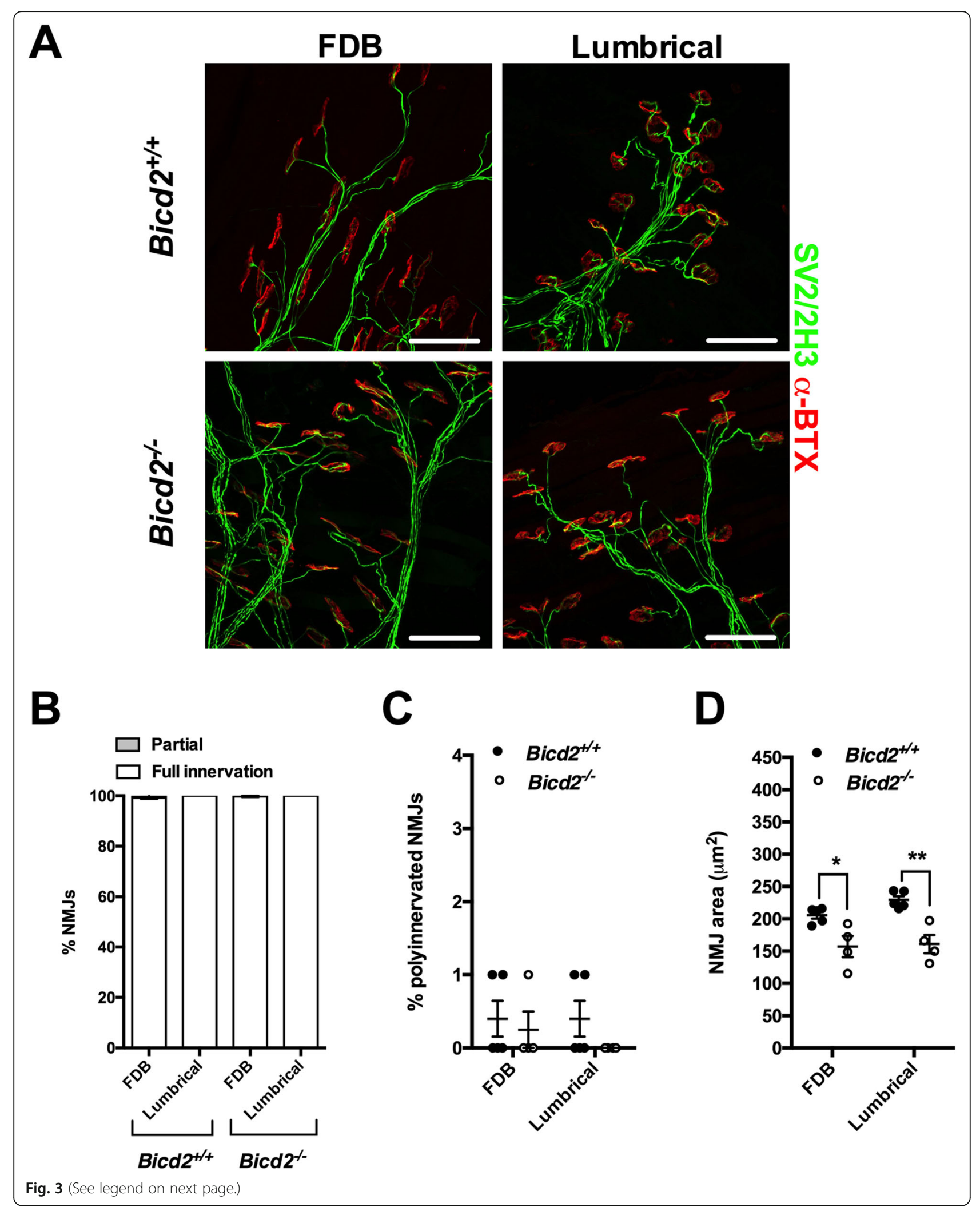


(See figure on previous page.)

Fig. 3 Normal NMJ analysis of Bicd2 ${ }^{-1-}$ mice at 21 days of age. a shows representative images of the NMJs of the FDB (flexor digitorum brevis) and feet lumbrical muscles stained with anti-SV2/2H3 antibodies (green) to visualise motor neurons and fluorescent alpha-bungarotoxin (red) to identify post-synaptic acetylcholine receptors on the muscle fibre surface. Scale bars $=50 \mu \mathrm{m}$. $\mathbf{b}$ shows the percentage of fully and partially innervated NMJs in $B i c d 2^{+/+}(n=4)$ and


the area of the NMJ (area occupied by each single AchR cluster) in the FDB and lumbrical muscles in Bicd2 ${ }^{+/+}$(mean 205 and $230 \mu m^{2}$, respectively; $n=4$ ) and Bicd2 ${ }^{-/-}$(mean 157 and $161 \mu \mathrm{m}^{2}$, respectively; $\mathrm{n}=4$ ) mice, (multiple $t$-tests corrected for multiple comparisons using the Holm-Sidak method, ${ }^{*} p=0.05$, ${ }^{*} p=0.004$ ). Error bars $=\mathrm{SEM}$. The normal NMJ analysis suggests that there is no active denervation in Bicd2 ${ }^{-1-}$ mice at 21 days of age

and instead contact muscle spindles. At three weeks, the distinction of motor neuron subtype based on axon diameter is unreliable [24]. To confirm whether the loss of L4 ventral axons at 3 weeks also correlated with a reduction in gamma motor neurons, we examined the total number of muscle spindles in the soleus muscle. The soleus muscle was chosen as it is a small muscle in which the total number of muscle spindles can be accurately quantified on serial transverse sections as previously described [20]. By staining for the SV2 and $2 \mathrm{H} 3$ antigens, muscle spindles can be identified, although gamma motor neuron efferents and 1a sensory afferents are indistinguishable. A comparison of the total number of muscle spindles revealed a $20 \%$ reduction in $B i c d 2^{-/-}$mice $(9.4 \pm 0.68)$ compared to wild type (12.5 \pm 0.29 , unpaired $t$-test $p=0.0065)$ (Fig. $4 \mathrm{~b})$. This correlates well with the reduction in smaller diameter motor axons at six weeks in Myod-Cre mice (Fig. 2d) and confirms that the motor axons lost at three weeks are also gamma motor neurons. The innervation pattern of the remaining muscle spindles was unperturbed, suggesting a developmental, as opposed to a degenerative process. Of note, there was no reduction in large diameter (presumed sensory 1a afferents) in the L4 dorsal nerve root of Bicd $2^{-1-}$ (Fig. 1d-e) and Myod-Cre mice (data not shown). It is possible that SV2 staining is specific to the gamma motor neuron endplate and does not stain for sensory 1a afferents that do not form synapses at the muscle spindle.

\section{Patient fibroblasts show evidence of impaired secretion}

Rab6, a small GTPase interacting with BICD2, is an established regulator of the secretory pathway $[29,30]$ and controls the flow of secreted proteins that are transported from the Golgi to the plasma membrane via a microtubuledependent process. As a consequence, loss of Rab6 results in a global reduction of protein secretion [22].

We therefore hypothesised that SMALED2-causing mutations in BICD2 result in an impairment of Rab6 function through its mislocalisation, impairing the targeting of secretory vesicles to the plasma membrane. This would explain why mutations in the N-terminal domain of BICD2, which enhance retrograde processive motility leading to accumulation of BICD2 at the centromere $[5,10]$, and C-terminal mutations that impair the recruitment of Rab6, but not dynein binding, to BICD2 cause an identical phenotype [31].

To verify this hypothesis, we performed an established VSV-G secretion assay [32] using fibroblasts from a patient with a $B I C D 2^{1189 \mathrm{~F}}$ SMALED2 mutation and from an agematched control [31]. These cells were transfected with a plasmid encoding for a temperature-sensitive mutant of

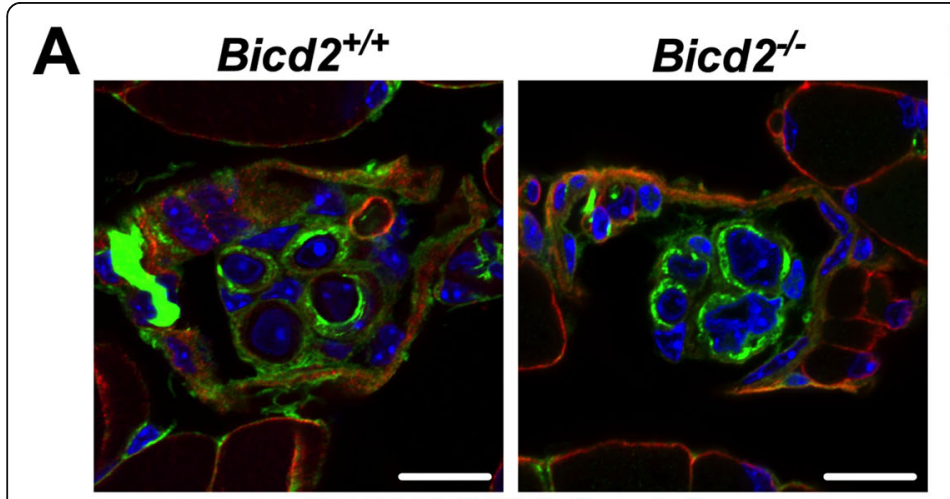

\section{DAPI SV2/2H3 Laminin}
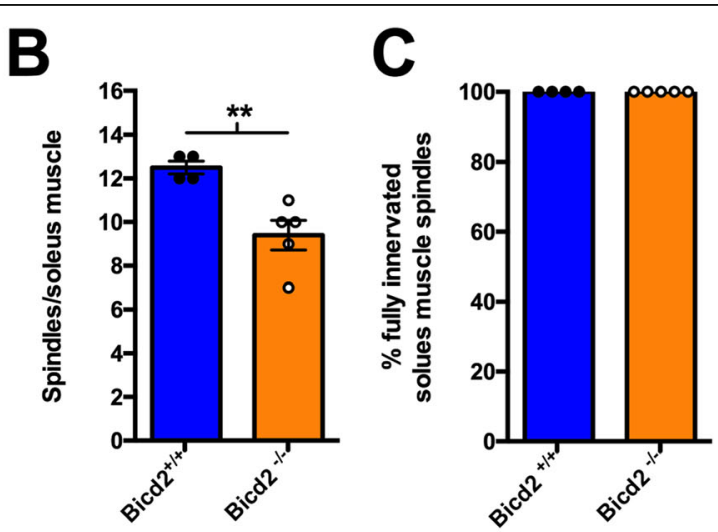

Fig. 4 Loss of muscle spindles in Bicd2 $2^{-/-}$mice at 21 days of age. (a) shows example images of a cross section through a muscle spindle in Bicd $2^{+/+}$and Bicd $2^{-/-}$mice stained for nuclei (DAPI, blue), the neuronal marker SV2/2H3 (green) and laminin (muscle membrane, red). Scale bars $=10 \mu m$. The concentric SV2/SH3 staining around laminin positive muscle fibres indicates a muscle spindle (b) shows the total number of muscle spindles in the soleus muscle of Bicd $2^{+/+}$and Bicd2 $2^{-/-}$mice, ${ }^{* *} p=0.0065$ (unpaired $t$-test, $\mathrm{n}=4-5$ ). (c) shows full innervation patterns in the muscle spindles of Bicd $2^{+/+}$and Bicd2 $2^{-/-}$mice. Error bars $=$ SEM. The loss of muscle spindles correlates with the loss of presumed gamma motor neurons in Bicd $2^{-/-}$compared to Bicd $2^{+/+}$mice 
vesicular stomatitis virus glycoprotein (ts0-45 VSV-G) tagged with GFP and incubated at $40^{\circ} \mathrm{C}$ for $14 \mathrm{~h}$. This high temperature treatment results in misfolding of ts $0-45$ VSV-G and its retention in the ER. Cooling of the cells to $32{ }^{\circ} \mathrm{C}$ allows the refolding of VSV-G and its targeting to the plasma membrane, which could then be quantified. This assay revealed a significant reduction in the rate of secretion of VSVG over time (Fig. 5b and c). Similar to previous experiments performed in Rab6 knockout cell lines [29, 32], secretion was significantly delayed, but not abolished. To confirm that this difference was not simply due to clonal differences, the accumulation of VSV-G at the plasma membrane at $240 \mathrm{~min}$ (the time point with the largest difference) was repeated for two additional, unrelated, age-matched controls and SMALED2 patients bearing the S107L and R501P mutations in BICD2. These additional experiments showed a significant reduction in VSV-G appearance on the plasma membrane at $240 \mathrm{~min}$ (Fig. 5c; control mean $=0.64 \pm 0.017, \quad n=3 ; \quad$ SMALED2 $=0.52 \pm$ $0.043, \mathrm{n}=3$; unpaired $t$-test $p=0.05$ ).

Since BICD2 and Rab6 have also been involved in COPI-independent transport from the Golgi to the ER
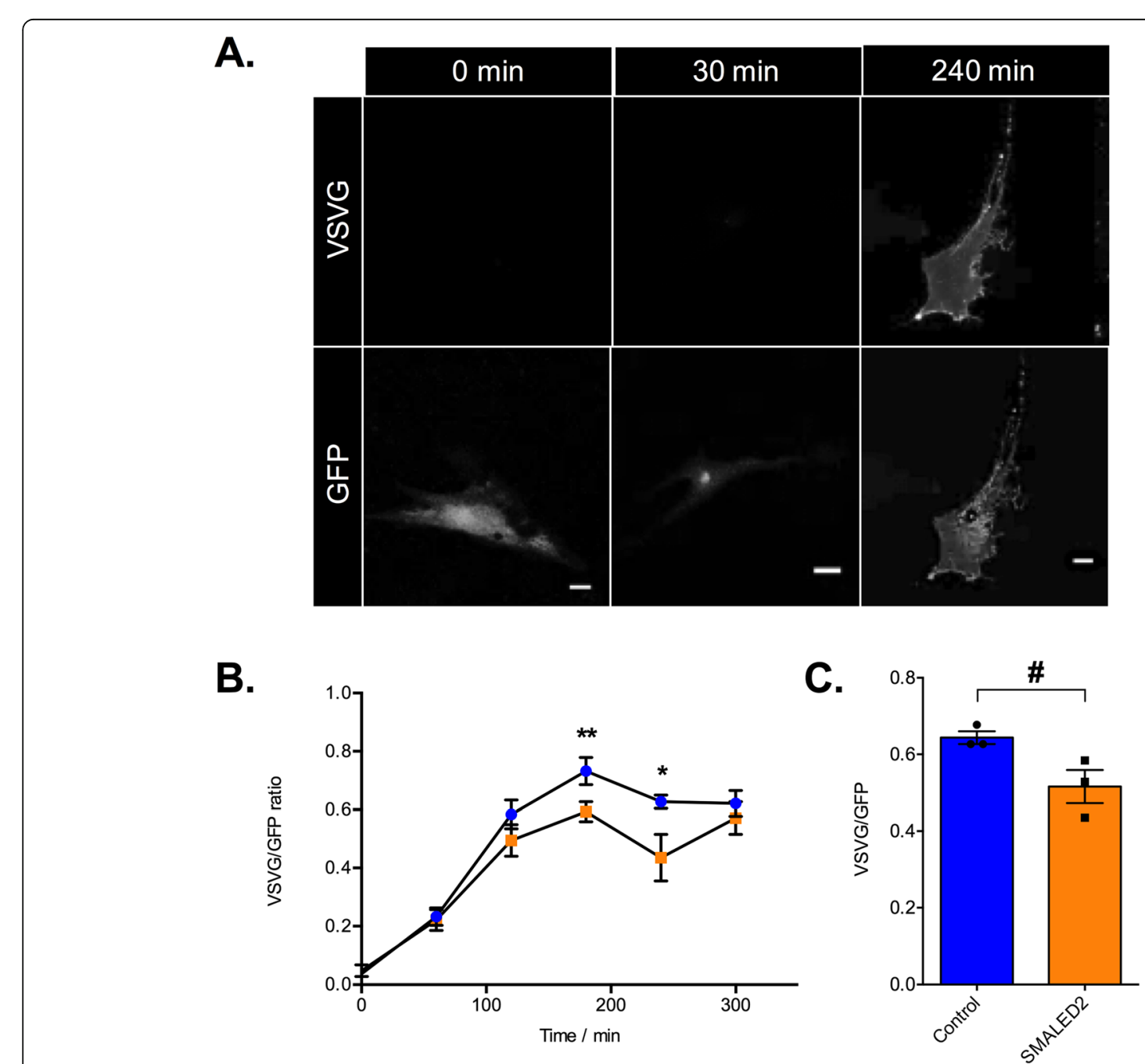

Fig. 5 SMALED2 patient fibroblasts show delayed VSV-G secretion compared to controls. $\mathbf{a}$ is an example of a human fibroblast transfected with a plasmid encoding for the thermo-sensitive-GFP vesicular stomatitis virus glycoprotein (tsO-45 VSV-G) at $32^{\circ} \mathrm{C}$. The time prior to fixation is indicated on the top; staining with an anti-VSV-G [8G5F11] against a surface epitope of VSV-G in non-permeabilised cells (top row of panels); GFP staining is shown in the bottom row. Scale bars $=20 \mu \mathrm{m}$. At 0 mins, all ts0-45 VSVG is retained within the ER with no surface staining. At 30mins all ts0-45 VSV-G has been trafficked to the Golgi. By 240 mins all ts045-VSV-g has been trafficked to the plasma membrane and is evident in both the GFP (bottom) and anti-VSV-G surface epitope antibody (top)_ panels. b Kinetics of VSV-G secretion in fibroblasts isolated from a patient with SMALED2 (I189F mutation, orange) and an age-matched control (blue) are quantified as the ratio of total surface VSV-G staining to total GFP. The $x$ axis shows the time in minutes at $32^{\circ} \mathrm{C}$ prior to fixation ( $n=10$ cells per condition; ${ }^{* *} p=0.008,{ }^{*} p=0.009$; multiple unpaired $t$-tests corrected for multiple comparisons using the Holm-Sidak method). $\mathbf{c}$ shows the average ratio of surface VSV-G to total GFP at $240 \mathrm{~min}$ for three independent healthy control and three unrelated SMALED2 (S107L, I189F and R501P) fibroblast cell lines ( $\#=0.052$, unpaired t-test). Error bars $=$ SEM. The impaired secretion in SMALED2 patient fibroblasts suggests that a similar impairment of secretion may be evident in the muscle of Bicd2 ${ }^{-1-}$ mice and SMALED2 patients 
[33, 34], we investigated the retrograde flow from the Golgi to the ER using galactosyltransferase-GFP in Brefeldin A-treated control and SMALED2 fibroblasts, but did not detect any significant difference between patient and control cells (Supplementary Figure 4A\&B).

In light of these results, we propose a non-cell autonomous mechanism of motor neuron loss in SMALED2, which is also likely to be applicable to SMALED caused by mutations in DYNC1H1 (Summarised in Fig. 6). We have shown that loss of BICD2 leads to death of motor neurons and that this is a non-cell autonomous process driven by the loss of BICD2 in muscle. Altogether, our results demonstrate that the $B I C D 2^{-/-}$mouse is a good model of SMALED2 as $B i c d 2^{-/}$mice and patients with severe SMALED2 mutations show similar clinical features including hydrocephalus, cerebellar hypoplasia and cortical migration defects $[13,14]$. A non-cell autonomous pathomechanism is also supported by a previous study demonstrating that the cerebellar hypoplasia in $B i c d 2^{-/-}$is driven by non-cell autonomous deficits arising in Bergmann glia [13].

Quantitative analyses of secretion in patient fibroblasts compared to controls shows only a modest secretion deficit, as found in Rab6 knockout models [22]. How such a delay in secretion causes a significant loss of motor function can be explained in light of the neurotrophin hypothesis in which a surplus of motor neurons compete for muscle- secreted neurotrophic factors for survival [24]. In this model, a reduction in the rate of secretion would yield a decrease in neurotrophin availability, driving an excess of motor neuron death. This hypothesis predicts that no progressive motor neuron degeneration should occur in SMALED2 and muscle-deficient BICD2 mice, as demonstrated in this work and elsewhere [31,35].

Our model may also explain why the loa, crawling and sprawling mouse models, which bear point mutations in Dync1h1 develop gamma motor neuron and sensory 1a afferent loss [25, 26, 36]. Both gamma motor neurons and 1a sensory afferents innervate muscle spindles and rely on muscle-derived neurotrophic factor secretion during early development. Therefore, an impairment of localised neurotrophin secretion from the muscle of dync1h1 mutant muscles would lead to a loss of gamma motor and sensory 1a neurons.

Our model does not align with previous results generated using a Drosophila model of SMALED2 [37], which led to the proposal that this pathology is determined by a cell autonomous effect of mutant BICD2 in motor neurons [37]. However, Drosophila have only a bicaudal D protein (BicD), unlike mice and humans, which express four bicaudal D proteins (BICD1, BICD2, BICDR1 and BICDR2) with different functions and interacting partners $[38,39]$. As such, the model of Martinez-Carrera is expected to be unable to



Fig. 6 A summary of the proposed non-cell autonomous mechanism in SMALED2. In normal muscle, BICD2 ensures the correct localisation of Rab6 at the trans Golgi surface, required for the efficient trafficking of secretory vesicles (containing neurotrophins) to the plasma membrane. In SMALED2 muscle, loss of BICD2 function impairs the localisation of Rab6 to the trans Golgi surface resulting in impaired trafficking of secretory vesicles to the plasma membrane and a reduction in neurotrophin release required for motor neuron survival 
differentiate between the effects of BICD1 and 2, which is crucial, as we have previously shown that BICD1 has an essential cell autonomous role in the sorting of signalling endosomes in motor neurons [40, 41]. Furthermore, Drosophila display important differences to mammals in the neurotrophin pathway at the NMJ [42].

Our model provides strong evidence in support of a non-cell autonomous mechanism of motor neuron loss in SMALED2 which involves impaired secretion of musclederived neurotrophins during development. However, a number of important questions still need to be addressed in future studies. The nature of the secreted musclederived neurotrophic factor(s) is still unclear as well as whether the phenotypes of mice with loss-of-function mutations in Bicd2 are similar to that of Bicd $2^{-/-}$mice. However, the most important point to clarify in future studies is likely to be the precise time point at which motor neuron loss occurs, which is important when considering future therapeutic interventions in SMALED2 patients.

\section{Supplementary information}

Supplementary information accompanies this paper at https://doi.org/10. 1186/s40478-020-00909-6.

Additional file 1: Supplementary Figures.

\section{Abbreviations}

BFA: Brefeldin A; BICD2: Bicaudal D2; SMALED: Spinal muscular atrophy lower extremity predominant; VSV: Vesicular stomatitis virus

\begin{abstract}
Acknowledgements
We are grateful to Professor Kathryn North and Dr. Emily Oates for providing patient fibroblasts, to the MRC Centre Biobank in London for provision of the patient fibroblast lines used in this study and to Pierpaolo Ala, technician in the Biobank. The Biobank is also supported by the National Institute for Health Research (NIHR) Great Ormond Street (GOS) Hospital Biomedical Research Centre. We are also grateful to Stéphanie Miserey-Lenkei for providing the GFP-VSV-G ts045 plasmid and for technical advice. We are also grateful to Professor Masayuki Murata for providing the GT-GFP plasmid. We are also grateful to Professor Rob Brownstone and Dr. Nadine SimonsWeidenmaier for providing the ChAT-Cre mice and to Dr. Stephane Nedelec for critical reading of the manuscript. The core support of MDUK to the UCL Neuromuscular Centre is also gratefully acknowledged.
\end{abstract}

\section{Authors' contributions}

A.M.R. wrote the draft of the manuscript and performed dissections, cell culture experiments and data analysis. A.M.R. and G.S. designed the experiments. J.N.S performed the NMJ, muscle spindle and DRG analyses. MG produced the semithin nerve root sections. C.H. provided the Bicd2 $2^{\text {loxP/loxP }}$ mice and F.M. and M.M.R. provided essential reagents. A.M.R., J.N.S. and G.S. revised the text and figures. The authors read and approved the final manuscript.

\section{Funding}

J.N.S. is funded by the Medical Research Council Career Development Award (MR/S006990/1). This work was supported by a Wellcome Trust Senior Investigator Award 107116/Z/15/Z (to G.S.), the European Union's Horizon 2020 Research and Innovation programme under grant agreement 739572 (to G.S.), a UK Dementia Research Institute Foundation award (to G.S.). and a Wellcome Trust Postdoctoral Fellowship for Clinicians (110043/Z/15/Z) (A.M.R).

\section{Availability of data and materials}

All data generated or analysed during this study are included in this published article [and its supplementary information files].

\section{Ethics approval and consent to participate}

SMALED2 patient and control fibroblasts were obtained with consent from the MRC CNMD Biobank, London which has been approved by the West London \& GTAC Research Ethics Committee (06/Q0406/33) and is licensed by the Human Tissue Authority (license number: 12198). Animal experimentation was performed under license from the United Kingdom Home Office in accordance with the Animals (Scientific Procedures) Act (1986), and approved by the University College London Queen Square Institute of Neurology ethics committee.

\section{Consent for publication}

Not applicable.

\section{Competing interests}

The authors declare that they have no competing interests.

\section{Author details}

${ }^{1} \mathrm{UCL}$ Queen Square Institute of Neurology, University College London, Queen Square, London WC1N 3BG, UK. ${ }^{2}$ UK Dementia Research Institute, University College London, London WC1E 6BT, UK. ${ }^{3}$ Dubowitz

Neuromuscular Centre and National Institute for Health Research Great Ormond Street Hospital Biomedical Research Centre, University College London Institute of Child Health, London WC1N 1EH, UK. ${ }^{4}$ Cell Biology, Neurobiology and Biophysics, Department of Biology, Utrecht University, Padualaan 8, $3584 \mathrm{CH}$ Utrecht, The Netherlands. ${ }^{5}$ Discoveries Centre for Regenerative and Precision Medicine, University College London Campus, London WC1N 3BG, UK.

Received: 29 February 2020 Accepted: 1 March 2020

Published online: 17 March 2020

\section{References}

1. Oates EC, Rossor AM, Hafezparast M, Gonzalez M, Speziani F, Macarthur DG et al (2013) Mutations in BICD2 cause dominant congenital spinal muscular atrophy and hereditary spastic paraplegia. Am J Hum Genet 92:965-973

2. Neveling K, Martinez-Carrera LA, Hölker I, Heister A, Verrips A, HosseiniBarkooie SM et al (2013) Mutations in BICD2, which encodes a golgin and important motor adaptor, cause congenital autosomal-dominant spinal muscular atrophy. Am J Hum Genet 92:946-954

3. Scoto M, Rossor AM, Harms MB, Cirak S, Calissano M, Robb S et al (2015) Novel mutations expand the clinical spectrum of DYNC1H1-associated spinal muscular atrophy. Neurology. 84:668-679

4. Harms MB, Ori-McKenney KM, Scoto M, Tuck EP, Bell S, Ma D et al (2012) Mutations in the tail domain of DYNC1H1 cause dominant spinal muscular atrophy. Neurology. 78:1714-1720

5. Peeters K, Litvinenko I, Asselbergh B, Almeida-Souza L, Chamova T, Geuens T et al (2013) Molecular defects in the motor adaptor BICD2 cause proximal spinal muscular atrophy with autosomal-dominant inheritance. Am J Hum Genet 92:955-964

6. Schiavo G, Greensmith L, Hafezparast M, Fisher EMC (2013) Cytoplasmic dynein heavy chain: the servant of many masters. Trends Neurosci 36:641-651

7. Klinman E, Holzbaur ELF (2018) Walking forward with Kinesin. Trends Neurosci 41 : 555-556

8. Sleigh JN, Rossor AM, Fellows AD, Tosolini AP, Schiavo G (2019) Axonal transport and neurological disease. Nat Rev Neurol 15:691-703

9. Hoang HT, Schlager MA, Carter AP, Bullock SL (2017) DYNC1H1 mutations associated with neurological diseases compromise processivity of dyneindynactin-cargo adaptor complexes. Proc Natl Acad Sci 114:E1597-E1606

10. Huynh W, Vale RD (2017) Disease-associated mutations in human BICD2 hyperactivate motility of dynein-dynactin. J Cell Biol 216:3051-3060

11. Hoogenraad CC, Akhmanova A, Howell SA, Dortland BR, De Zeeuw Cl, Willemsen $R$ et al (2001) Mammalian golgi-associated Bicaudal-D2 functions in the dynein-dynactin pathway by interacting with these complexes. Embo J 20:4041-4054

12. Storbeck M, Horsberg Eriksen B, Unger A, Hölker I, Aukrust I, Martínez-Carrera LA et al (2017) Phenotypic extremes of BICD2-opathies: from lethal, congenital muscular atrophy with arthrogryposis to asymptomatic with subclinical features. Eur J Hum Genet 25:1040-1048

13. Jaarsma D, van den Berg R, Wulf PS, van Erp S, Keijzer N, Schlager MA et al (2014) A role for Bicaudal-D2 in radial cerebellar granule cell migration. Nat Commun 5:3411 
14. Ravenscroft G, Di Donato N, Hahn G, Davis MR, Craven PD, Poke G et al (2016) Recurrent de novo BICD2 mutation associated with arthrogryposis multiplex congenita and bilateral perisylvian polymicrogyria. Neuromuscul Disord 26:744-748

15. Otto C, Fuchs I, Kauselmann G, Kern H, Zevnik B, Andreasen P et al (2009) GPR30 does not mediate estrogenic responses in reproductive organs in mice. Biol Reprod 80:34-41

16. Kanisicak O, Mendez JJ, Yamamoto S, Yamamoto M, Goldhamer DJ (2009) Progenitors of skeletal muscle satellite cells express the muscle determination gene, MyoD. Dev Biol 332:131-141

17. Rossi J, Balthasar N, Olson D, Scott M, Berglund E, Lee CE et al (2011) Melanocortin-4 receptors expressed by cholinergic neurons regulate energy balance and glucose homeostasis. Cell Metab 13:195-204

18. Mayeuf-Louchart A, Hardy D, Thorel Q, Roux P, Gueniot L, Briand D et al (2018) MuscleJ: a high-content analysis method to study skeletal muscle with a new Fiji tool. Skelet Muscle 8:25

19. Sleigh JN, Burgess RW, Gillingwater TH, Cader MZ (2014) Morphological analysis of neuromuscular junction development and degeneration in rodent lumbrical muscles. J Neurosci Methods 227:159-165

20. Sleigh JN, Dawes JM, West SJ, Wei N, Spaulding EL, Gómez-Martín A et al (2017) Trk receptor signaling and sensory neuron fate are perturbed in human neuropathy caused by gars mutations. Proc Natl Acad Sci U S A 114:E3324-E3333

21. Sleigh JN, Weir GA, Schiavo G (2016) A simple, step-by-step dissection protocol for the rapid isolation of mouse dorsal root ganglia. BMC Res Notes 9:82

22. Bardin S, Miserey-Lenkei S, Hurbain I, Garcia-Castillo D, Raposo G, Goud B (2015) Phenotypic characterisation of RAB6A knockout mouse embryonic fibroblasts. Biol Cell 107:427-439

23. Kablar B, Belliveau AC (2005) Presence of neurotrophic factors in skeletal muscle correlates with survival of spinal cord motor neurons. Dev Dyn 234:659-669

24. Kanning KC, Kaplan A, Henderson CE (2010) Motor neuron diversity in development and disease. Annu Rev Neurosci 33:409-440

25. Hafezparast M, Klocke R, Ruhrberg C, Marquardt A, Ahmad-Annuar A, Bowen $S$ et al (2003) Mutations in dynein link motor neuron degeneration to defects in retrograde transport. Science. 300:808-812

26. Ilieva HS, Yamanaka K, Malkmus S, Kakinohana O, Yaksh T, Marsala M et al (2008) Mutant dynein (Loa) triggers proprioceptive axon loss that extends survival only in the SOD1 ALS model with highest motor neuron death. Proc Natl Acad Sci U S A 105:12599-12604

27. Unger A, Dekomien G, Güttsches A, Dreps T, Kley R, Tegenthoff M et al (2016) Expanding the phenotype of BICD2 mutations toward skeletal muscle involvement. Neurology. 87:2235-2243

28. Gould TW, Yonemura S, Oppenheim RW, Ohmori S, Enomoto H (2008) The neurotrophic effects of glial cell line-derived neurotrophic factor on spinal motoneurons are restricted to fusimotor subtypes. J Neurosci 28:2131-2146

29. Grigoriev I, Splinter D, Keijzer N, Wulf PS, Demmers J, Ohtsuka T et al (2007) Rab6 regulates transport and targeting of Exocytotic carriers. Dev Cell 13:305-314

30. Fourriere L, Kasri A, Gareil N, Bardin S, Bousquet H, Pereira D et al (2019) RAB6 and microtubules restrict protein secretion to focal adhesions. J Cell Biol 218:2215-2231

31. Rossor AM, Oates EC, Salter HK, Liu Y, Murphy SM, Schule R et al (2015) Phenotypic and molecular insights into spinal muscular atrophy due to mutations in BICD2. Brain. 138:293-310

32. Miserey-Lenkei S, Chalancon G, Bardin S, Formstecher E, Goud B, Echard A (2010) Rab and actomyosin-dependent fission of transport vesicles at the Golgi complex. Nat Cell Biol 12:645-654

33. White J, Johannes L, Mallard F, Girod A, Grill S, Reinsch S et al (1999) Rab6 coordinates a novel Golgi to ER retrograde transport pathway in live cells. J Cell Biol 147:743-760

34. Matanis T, Akhmanova A, Wulf P, Del Nery E, Weide T, Stepanova T et al (2002) Bicaudal-D regulates COPI-independent Golgi-ER transport by recruiting the dynein-dynactin motor complex. Nat Cell Biol 4:986-992

35. Oates EC, Reddel S, Rodriguez ML, Gandolfo LC, Bahlo M, Hawke SH et al (2012) Autosomal dominant congenital spinal muscular atrophy: a true form of spinal muscular atrophy caused by early loss of anterior horn cells. Brain. 135:1714-1723

36. Chen X-J, Levedakou EN, Millen KJ, Wollmann RL, Soliven B, Popko B (2007) Proprioceptive sensory neuropathy in mice with a mutation in the cytoplasmic dynein heavy chain 1 gene. J Neurosci 27:14515-14524

37. Martinez Carrera LA, Gabriel E, Donohoe CD, Hölker I, Mariappan A, Storbeck M et al (2018) Novel insights into SMALED2: BICD2 mutations increase microtubule stability and cause defects in axonal and NMJ development. Hum Mol Genet 27:1772-1784

38. Hoogenraad CC, Akhmanova A (2016) Bicaudal D family of motor adaptors: linking dynein motility to cargo binding. Trends Cell Biol 26:327-340

39. Terenzio M, Schiavo G (2010) The more, the better: the BICD family gets bigger. EMBO J 29:1625-1626

40. Terenzio M, Golding M, Russell MRG, Wicher KB, Rosewell I, Spencer-Dene B et al (2014) Bicaudal-D1 regulates the intracellular sorting and signalling of neurotrophin receptors. EMBO J 33:1-17

41. Terenzio M, Golding M, Schiavo G (2014) siRNA screen of ES cell-derived motor neurons identifies novel regulators of tetanus toxin and neurotrophin receptor trafficking. Front Cell Neurosci 8:140

42. Sutcliffe B, Forero MG, Zhu B, Robinson IM, Hidalgo A (2013) Neuron-type specific functions of DNT1, DNT2 and Spz at the Drosophila neuromuscular junction. PLoS One 8:e75902

\section{Publisher's Note}

Springer Nature remains neutral with regard to jurisdictional claims in published maps and institutional affiliations.

Ready to submit your research? Choose BMC and benefit from:

- fast, convenient online submission

- thorough peer review by experienced researchers in your field

- rapid publication on acceptance

- support for research data, including large and complex data types

- gold Open Access which fosters wider collaboration and increased citations

- maximum visibility for your research: over $100 \mathrm{M}$ website views per year

At BMC, research is always in progress.

Learn more biomedcentral.com/submissions 